BULLETIN Bulletin hispanique

HISPANIQUE Université Michel de Montaigne Bordeaux

$115-2$ | 2013

Les traductions vieillissent-elles?

\title{
Les verbes italiens stare, sedere et giacere
}

analyse de cinq traductions françaises du Décaméron de Jean Boccace

Damien Zalio

\section{(2) OpenEdition}

Journals

Édition électronique

URL : http://journals.openedition.org/bulletinhispanique/2700

DOI : $10.4000 /$ bulletinhispanique. 2700

ISSN : $1775-3821$

Éditeur

Presses universitaires de Bordeaux

Édition imprimée

Date de publication : 28 décembre 2013

Pagination : 507-528

ISBN : 978-2-86781-908-7

ISSN : 0007-4640

Référence électronique

Damien Zalio, "Les verbes italiens stare, sedere et giacere », Bulletin hispanique [En ligne], 115-2 | 2013,

mis en ligne le 14 février 2017, consulté le 19 avril 2019. URL : http://journals.openedition.org/

bulletinhispanique/2700; DOI : 10.4000/bulletinhispanique.2700 


\title{
Les verbes italiens stare, sedere et giacere: analyse de cinq traductions françaises du Décaméron de Jean Boccace
}

\author{
Damien ZaLio \\ Université Paris-Sorbonne
}

Les verbes de position latins stare, sedere et iacere ont tous trouvé un prolongement dans les langues romanes, et l'italien offre à cet égard une stabilité remarquable. Par l'étude de cinq traductions françaises du Décaméron, il s'agira d'essayer de distinguer ce qui revient à la référence de ce qui est du domaine du signe, ainsi que de vérifier si ces deux entités linguistiques vieillissent.

Mots-clés : verbe, sémantique, signifiant, italien.

Los verbos latinos de posición stare, sedere $e$ iacere han pervivido todos en las lenguas románicas y al respecto, el italiano ofrece una estabilidad notable. Mediante el estudio de cinco traducciones francesas del Decamerón, intentaremos distinguir lo que pertenece a la referencia de lo que es del ámbito del signo, asi como averiguar si esas dos entidades lingüisticas envejecen.

Palabras clave : verbo, semántica, significante, italiano.

All three Latin verbs of position stare, sedere and iacere have persisted in Romance languages. In this regard, Italian presents a striking stability. By studying five French translations of the Decameron, we will try to discriminate what pertains to reference from what belongs to the sign field, and to see if these two linguistic entities do age.

Keywords : verb, semantics, signifier, Italian.

\section{INTRODUCTION}

Les traductions vieillissent-elles ? Voilà une question gigogne. Si l'on considère comme acquis que toute langue vieillit, tout travail se servant de ce code sémiologique comme support de communication est a priori appelé à Bulletin Hispanique, Tome 115, n 2 - décembre 2013 - p. 507-528. 
vieillir lui aussi. Les traductions vieillissent-elles donc d'elles-mêmes car elles sont le fruit d'une époque révolue, ou peuvent-elles également vieillir le textesource par les prises de position des traducteurs et des éditeurs ? C'est cette deuxième question que nous tenterons ici de développer.

Les verbes dits " premiers » ont probablement une place à part dans l'exercice de la traduction. De par leur très forte fréquence, ils ne posent pas directement problème, et leur rendu dans la langue cible s'exécute certainement de façon spontanée. Pourtant, après examen de divers procédés de traduction des verbes italiens stare, sedere et giacere dans le Décaméron de Jean Boccace, il apparaît que certains mécanismes latents sont à l'œuvre, même pour les deux langues typologiquement apparentées que sont l'italien et le français.

À travers le prisme des dérivés des verbes latins stare, sedere et iacere, nous proposons de comparer cinq traductions françaises du Décaméron, dont la première (début du XV $\mathrm{XV}^{\mathrm{e}}$ siècle) et la dernière en date (2006). Nous émaillerons notre exposé d'exemples extraits de notre corpus, constitué de 125 occurrences des trois verbes en question et de leurs traductions respectives. Dans un premier temps, il s'agira de présenter la réception du livre des cent nouvelles en France et la fortune de quelques-unes de ses traductions, et de proposer un bref aperçu des descendants italiens et français des étymons latins. Nous consacrerons la troisième partie de ce travail à l'observation du corpus, avant de dresser un inventaire des capacités référentielles qu'autorise chacun des signes considérés et des constantes repérées dans les traductions françaises.

\section{Le DéCAMÉRON et SA RÉCEPTION en France}

Comédie humaine considérée comme le pendant terrestre de la Divine Comédie de Dante Alighieri, le Décaméron de Jean Boccace, rédigé entre 1349 et 1351, est le premier recueil de nouvelles du genre en Occident. Il se caractérise par la multiplicité des situations qu'il dépeint, des plus sérieuses aux plus cocasses, mais également par la variété des lieux de l'action, la diversité des thèmes abordés ainsi que par la multitude de classes sociales de ses personnages (avec toutefois une prédominance de la classe marchande, émergente en Italie à cette époque). Le style y est à la fois soutenu, voire latinisant, mais comporte également un registre parfois plus familier. C'est à ce matériau narratif et linguistique polymorphe et polyphonique, brossant un portrait minutieux de la société italienne du Trecento - mais aussi, parfois, d'époques antérieures et de rivages plus lointains - que le traducteur doit se confronter. Cela, qu'il soit contemporain de Boccace ou que plus de six siècles les séparent.

La première traduction française du Décaméron (1411-1414) est l'œuvre de Laurent de Premierfait. Il s'agit cependant d'une traduction-relais depuis le latin vers le français d'après une première traduction, effectuée cette fois-ci de l'italien vers le latin par le cordelier Antoine d'Arezzo. Cette version connaîtra un certain succès puisqu' elle sera copiée et rééditée jusqu'au XVI ${ }^{e}$ siècle, période où « la traduction, peu accessible parce que présentant un texte archaïsant ou 
méconnaissable parce que continuellement réécrite, ne trouve plus grâce aux yeux des lecteurs et des éditeurs ${ }^{1}$.

La traduction d'Antoine le Maçon (1545), censée dépoussiérer la première, connaît alors une grande fortune. Quant à l' " infidèle » d'Antoine de Sabatier de Castres, elle sera réimprimée seize fois, de 1779 à 1929, et celle de Francisque Reynard (1879) est tenue pour " parfaitement intelligible pour le lecteur moderne et $[. .$.$] certainement la plus exacte de celles [\mathrm{du}$ XIX $]$ siècle $»^{2}$.

Viennent ensuite les traductions des $\mathrm{XX}^{\mathrm{e}}$ et $\mathrm{XXI}^{\mathrm{e}}$ siècles. Les préfaces des traducteurs nous offrent un précieux témoignage sur leurs intentions de départ. Pour Jean Bourciez par exemple, dont le travail date de 1952, l'objectif et l'utilité de son travail ne laissent aucun doute:

Dans les lignes qui précèdent, et qui s'adressent à des Français, je n'avais rien à dire du style même de Boccace. Si l'on veut bien comparer le texte du Décaméron et la traduction qui va suivre, on s'apercevra tout de suite d'une certaine disparate, que mon devoir est de signaler. Boccace écrit dans la langue cléricale du quatorzième siècle, arrondie et repétrie à l'imitation de Cicéron et de Tite-Live. Il ne saurait être question de conserver cette forme, qui nous ramènerait au moins à l'époque de Rabelais. Il s'ensuit que les phrases, trop longues pour notre goût, ont dû être brisées et tronçonnées. J'ai maintenu, dans une certaine mesure, le tour de quelques passages oratoires. C'est tout ce que me permettaient, je crois, les ressources actuelles de notre langue. Cette transformation affecte-t-elle la teneur du livre ? J'ose espérer que non ${ }^{3}$.

Le choix est fait, justifié au lecteur et assumé. Il s'agit, dans ce « cahier des charges » cohérent, d'une traduction revisitée.

\section{STARE, SEDERE, GIACERE, VERBES DE POSITION}

Le latin possédait trois verbes de position principaux : stare ('se tenir debout', 's'arrêter'), sedere ('être assis', 'siéger', 'être arrêté dans l'esprit') et iacere ('être couché, 'se trouver en position horizontale').

\section{II.1 Italien}

Les trois verbes italiens stare, sedere et giacere proviennent respectivement des verbes latins sus-cités. À l'époque de Boccace, dans le système toscan du $\mathrm{XIV}^{\mathrm{e}}$ siècle, ils font partie de ces quelques verbes pouvant recevoir un pronom réfléchi, et ce, apparemment, de manière indifférente. Pour un même verbe, deux formes sont alors possibles :

1. Giuseppe Di Stefano, «Introduction », dans Giuseppe Di Stefano (dir.), Boccace, Décaméron, traduction de Laurent de Premierfait (1411-1414), Montréal, CERES, 1998, p. XXVI.

2. Vittore Branca, "Note pour le texte et la traduction ", dans Francisque Reynard (trad.), Le Décaméron. Édition présentée et annotée par Vittore Branca, Paris, Le club français du livre, p. XLVIII.

3. Jean Bourciez, "Boccace et le Décaméron", dans Jean Bourciez (trad.), Le Décaméron. Traduction nouvelle de Jean Bourciez, Paris, Garnier Frères, 1952, pp. XI-XII. C'est nous qui soulignons. 
Stare : starsi

Sedere : sedersi

Giacere : giacersi

La forme réfléchie n'est aujourd'hui possible qu'avec sedere et stare, si et seulement si ce dernier est accompagné du pronom adverbial ne (starsene). L'on peut donc considérer que l'italien moderne s'est privé de certaines capacités expressives en renonçant à laisser au locuteur le choix de sa visée de discours. En somme, pour ce cas de syntaxe, la Langue commande au Discours et choisit pour le sujet parlant, lequel jouit aujourd'hui d'une liberté moindre puisque la pronominalisation est désormais limitée à deux verbes seulement.

À côté de leur emploi dit absolu, ces verbes ont également la possibilité de s'employer en tant que copules. Ainsi, les phrases attributives sedere stupefatto, stare infermo ou giacere infermo sont attestées dans notre corpus.

\section{2 Français}

Si le français contemporain opère un mélange - être contient en un seul paradigme des formes dérivées de esse (i.e. je suis, je fus) et d'autres dérivées de stare (i.e. jétais, étant) -, l'ancien français possédait en revanche deux verbes bien distincts, même à l'infinitif. Estre, attesté dès le XIe siècle, provenant des deux étymons, signifie 'exister', mais aussi 'se trouver', 'demeurer', en plus d'indiquer un rapport de propriété. Ester, attesté un siècle plus tard, provient uniquement de stare, possède une extension moindre et signifie 'être debout', 's'arrêter' ou 'demeurer'. Ce verbe apparaît aujourd'hui, uniquement à l'infinitif, dans la locution juridique " ester en justice».

De son côté, l'étymon sedere en français a donné naissance à seoir et asseoir. Seoir est attesté dès le $\mathrm{X}^{\mathrm{e}}$ siècle ${ }^{4}$ et asseoir apparait d'abord sous rection transitive (fin du $\mathrm{X}^{\mathrm{e}}$ siècle), puis sous rection pronominale (mi-XI' siècle). Le second peut s'employer aussi bien transitivement qu'intransitivement tandis que le premier n'est qu'intransitif et défectif ${ }^{5}$. Le français asseoir a donc connu, dans une certaine mesure, l'évolution inverse à celle de son homologue italien seder(si): d'asseoir, on est passé à la possibilité de pronominaliser le verbe avec s'asseoir.

4. D’après le Trésor de la Langue Française Informatisé, seoir est considéré comme vieilli dès le XVII' siècle.

5. Seoir se conjugue principalement au mode quasi-nominal - infinitif, gérondif, participe " passé » et participe présent -, aux personnes de rang 3 de l'indicatif et du subjonctif présent, de l'indicatif imparfait, du futur et du conditionnel. Les formes composées sont inexistantes.

6. Il convient cependant de préciser que cette pronominalisation possible des verbes italiens cités ci-dessus est également caractéristique de l'ancien français : «En ancien français, beaucoup de verbes intransitifs, surtout les verbes de mouvement et de situation, pouvaient s'adjoindre le pronom réfléchi : on disait s'avaler, se demourer, s'ester, siissir, s'entrer, se gésir, se monter, se repairer, se seoir, se venir, etc. " ; cf. Joe Larochette, "Les aspects verbaux en espagnol moderne ", Revue belge de philologie et d'histoire, $\mathrm{n}^{\circ} 23,1944$, p. 42. 


\section{II.3 Un vieillissement à deux vitesses}

Mis à part l'éventuelle pronominalisation de stare et de giacere à date ancienne, aucune divergence notable n'est à déclarer entre l'état ancien et l'état contemporain de l'italien. À la lecture, un italophone connaisseur pourra détecter le si de starsi ou de giacersi comme un archaïsme, tandis que celui qui n'est pas sensibilisé à ces questions verra dans ce clitique une coquille ou une licence de l'auteur.

Le vieillissement interne se situe donc au niveau des verbes français, dont les paradigmes ont connu une très forte érosion. Mais ce phénomène a trouvé compensation dans la fréquence croissante de s'asseoir et de formes analytiques telles qu'être assis, être couché, être étendu, et être debout, par exemple, en face d'une rection transitive (asseoir et coucher, par exemple), rection que l'italien n’a développée ni pour stare ni pour sedere ni pour giacere, tous résolument intransitifs .

Si l'on en vient maintenant au français contemporain, les traductions les plus orthonymiques ${ }^{8}$ de stare, sedersi, sedere et giacere sont très certainement, respectivement, rester, être assis, s'asseoir et être couché. Seoir a été écarté au fur et à mesure, se déféctivisant au profit de asseoir.

\section{EXAMEN DU CORPUS}

\section{III.1 Sedere}

\section{III.1.1 Sedere in terra}

Observons les exemples suivants, où sedere précède ce circonstant de lieu dans le texte-source :

(1 a) E come levato fu la mattina, lasciato il suo fante, quando tempo gli parve, solo se n'andò verso la casa della sua donna. E per ventura trovata la porta aperta, entrò dentro e vide la sua donna sedere in terra in una saletta terrena che ivi era, ed era tutta piena di lagrime e d'amaritudine [...].

(Giovanni Boccaccio (c. 1349-1351), Decameron (sous la dir. de Cesare Segre), Milan, Mursia, 1966, p. 375).

7. Néanmoins, leurs pendants " référentiels " transitifs abondent. Selon les situations expérientielles à évoquer, l'italien recourt alors : 1) à une construction factitive (i.e. asseoir les enfants : mettere a sedere/far sedere $i$ bambini), 2) à un tout autre verbe transitif (i.e. asseoir sa théorie sur les faits : basarelfondarelconsolidare la propria teoria sui fatti) 3) à une forme périphrastique (i.e. coucher un enfant: mettere a letto un bambino; coucher une bouteille: stendere/poggiare una bottiglia a terra). Ce sont là quelques exemples parmi tant d'autres.

8. Terme forgé par Bernard Pottier : «[...] les entités ont une désignation privilégiée, immédiate, dans une situation, un environnement bien déterminé [...]. La céphalée sera l'orthonyme pour le médecin, le mal de tête pour le malade "; cf. Bernard Pottier, Sémantique générale [1992], 2011, Paris, PUF, p. 123. 
(1 b) Après ce que Tedald fut au matin levez, il laissa son varlet, et quant bon lui sambla, il ala en l'ostel de s'amie. Il qui d'adventure trouva la porte ouverte, entra dedans la maison et vit s'amie seant a terre en ung petit solier qui la estoit. Elle fut pleine de larmes et de amertume.

(Giuseppe di Stefano (dir.), Laurent de Premierfait (trad.), Boccace, Décaméron, traduction de Laurent de Premierfait (1411-1414), Montréal, CERES, 1998, p. 375)

(1 c) Laissant son domestique au logis, il va droit à la maison de la dame, pour s'informer si elle l'habite encore. Il trouve la porte de l'allée ouverte, et entre sans difficulté dans une petite salle basse, où il voit son ancienne maitresse dans le plus triste état. Elle sanglotait et était étendue sur le carreau, qu'elle inondait de ses larmes.

(Antoine Sabatier de Castres (trad.), Le Décaméron (1779), groupe Ebooks libres et gratuits, 2007, p. 251)

(1 d) Il se lève au matin, laisse son domestique et, quand le moment lui parut opportun, se dirige seul vers la maison de sa maitresse. Il eut la chance de trouver la porte ouverte. Il entre, il voit la dame, assise à même le sol, dans un méchant réduit qui n'était pas carrelé.

(Jean Bourciez (trad.), Le Décaméron. Traduction nouvelle de Jean Bourciez, Paris, Garnier Frères, 1952, p. 221)

(1 e) Le matin, quand il fut levé, à l'heure qui lui parut convenir, il s'en alla seul à la maison de sa dame. Et ayant trouvé la porte ouverte par un heureux hasard, il entra et vit sa dame assise, là, par terre, dans une petite pièce du rez-de-chaussée, ruisselant de larmes et brisée par la douleur.

(Christian Bec (dir.), Marthe Dozon (trad.), Catherine Guimbard (trad.), Marc Scialom (trad.), Décaméron, Paris, Librairie Générale Française, 1994, p. 280)

(1 f) Et lorsqu'au matin il se fut levé, il laissa là son serviteur et, quand bon lui sembla, se dirigea seul vers la maison de sa dame. Et d'aventure ayant trouvé la porte ouverte, il entra et vit dans une petite salle du rez-de-chaussée sa dame assise par terre et toute pleine de larmes et d'affliction.

(Giovanni Clerico (trad.), Le Décaméron, Paris, Gallimard, 2006, pp. 295-296)

Ces exemples posent le problème de la conceptualisation temporelle que renferme, et donc autorise ou interdit, chaque forme de langue. Pour une même situation référentielle (ici, 'se trouver en position assise, par terre'), il est possible de recourir à diverses imbrications syntaxiques. Boccace a choisi sedere in terra; il eût pu tout aussi bien écrire essere seduto in terra ou stare seduto in terra, 
cela n'aurait rien changé à la référenciation. Ce qui diffère, c'est le découpage temporel que propose chaque forme. Ainsi, en chronologie notionnelle, sedere, apportant la représentation d'une opération non encore amorcée, est antérieur à seduto, qui nous donne l'image de cette opération à son état achevé. Essere, hyperonyme de stare, lui est aussi antérieur. Soit schématiquement :

\begin{tabular}{c|c|c}
\multicolumn{3}{c}{ chronologie notionnelle } \\
\hline $\begin{array}{c}1 \\
\text { Sedere }\end{array}$ & 2 & 3 \\
essere seduto & stare seduto
\end{tabular}

En fonction des découpages chronologiques possibles offerts par chaque signe, le traducteur s'accommode de ce qu'il juge le plus opportun de donner à voir au lecteur. Comme l'affirme Marie-France Delport, pour toutes les traductions possibles d'un même vocable ou syntagme,

Un seul événement est en cause, et donc une seule date, un repère unique en chronologie d'expérience. Mais en chronologie de raison, l'implication réciproque des deux conceptualisations s'ordonne [...]. Au lieu donc de traduire TD [le Texte de Départ] au plus près, le traducteur s'est forgé, à la lecture de TD, la représentation d'un événement expérienciel et, au moment de produire TA [le Texte d'Arrivée], jouant sur le rapport d'implication qu'on a énoncé, dit l'avant quand TD disait l'après ou viceversa. ${ }^{9}$

Mais ce choix peut-être libre ou contraint. Ainsi, le français d'aujourd'hui, pour une même situation d'expérience, est incapable d'opérer un tel découpage sans chercher en-dehors des formes verbales simples son moyen d'expression. On obtient donc la phrase attributive être assis. Le moyen français, comme on le voit dans l'exemple de Premierfait, possédait en revanche une possibilité de découpage un peu plus complexe :

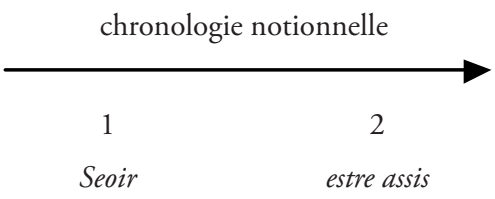

Possibilité était donc offerte à Laurent de Premierfait d'effectuer un choix : il a privilégié la représentation d'une opération commencée mais non achevée avec le gérondif séant, ce qui correspond à l'italien sedendo, et non celle du résultat, estre assis. Si le premier traducteur use de sa liberté dans les limites qu'autorise le système, Sabatier de Castres les outrepasse, préférant être étendu à être assis. Rien ne se garde, si ce n'est l'idée d'une position corporelle précise de l'être en question. Tous les traducteurs suivants ont recouru à assis, car il ne

9. Marie-France Delport, "Les horloges du traducteur ", dans Jean-Claude Chevalier, Marie-France Delport, L'horlogerie de Saint Jérôme. Problèmes linguistiques de la traduction, Paris, L'Harmattan, 1995, pp. 13-14. 
leur est désormais possible que d'exprimer l'après de sedere, ou de s'asseoir, si l'on préfere. Aux yeux d'un lecteur du XXI ${ }^{e}$ siècle, la traduction de Premierfait en ressort donc nécessairement vieille.

\section{III.1.2 Sedere appresso}

Poursuivons nos observations avec les occurrences de la cornice, ce récitcadre présent en-dehors de chaque nouvelle. Très souvent, et systématiquement lors de la première journée, le passage de relais entre les conteurs est évoqué de la façon suivante :

(2 a) La novella di Panfilo fu in parte risa e tutta commendata dalle donne: la quale diligentemente ascoltata e al suo fine essendo venuta, sedendo appresso di lui Neifle, le comandò la reina che, una dicendone, lordine dello incominciamento sollazzo seguisse.

$$
\text { (Giovanni Boccaccio, op. cit., p. 57) }
$$

(3 a) Già si tacea Filomena, dalla sua novella espedita, quando Dioneo, che appresso di lei sedeva, senza aspettare dalla reina altro comandamento, conoscendo già per l'ordine cominciato che a lui toccava il dover dire, in cotal guisa cominciò a parlare[.]

$$
\text { (Ibid., p. 63) }
$$

(4 a) Emilia, la quale appresso Fiammetta sedea, essendo già stato da tutte commendato il valore e il leggiadro gastigamento della marchesana fatto al re di Francia, come alla sua reina piacque, baldanzosamente a dire cominciò[.]

$$
\text { (Ibid., p. 69) }
$$

(5 a) Sedeva appresso Filostrato Lauretta, la quale, poscia che udito ebbe lodare la 'ndustria di Bergamino [...], senza alcun commandamento aspettare, piacevolmente cosí cominciò a parlare[.]

$$
\text { (Ibid., p. 75) }
$$

Ici, la position physique des jeunes hommes et femmes est explicitée. Il est dit des personnages à qui le tour vient de conter une nouvelle qu'ils sont à côté de celui qui vient d'en achever une, et qu'ils le sont en position assise : siedono. Comparons maintenant les traductions :

(2 b) La nouvelle premiere, racomptee par Pamphile, fut cause de riz et d'esbatemant et si fut toute louee de par les dames. Si tost que elle fut diligemmant ouye et finee, a Neiphile, qui seoit pres de Pamphile, la royne commenda que, en disend une nouvelle, elle poursuivist l'esbatemant commencié.

$$
\text { (Laurent de Premierfait, op. cit., p. 54) }
$$

(3 b) Ja se taisoit Philomene, despeschee de sa nouvelle, quant Dyonee, qui pres de elle seoit, sanz attendre aultre mandemant de la royne que selon l'ordonnance ja commancee il lui convenoit dire sa nouvelle, commança ainsi parler[.] 
(4 b) Dame Emilie, qui seoit pres de Flammete, commença ainsi parler au plaisir de la royne, après ce que recommandez furent de par totues les sept dames la valeur et gracieux chastoiemant que la marquise monstra en pou de paroles au roy de France, et dist ainsi Emilie[.]

$$
\text { (Ibid., p. 79) }
$$

(5 b) Dame Laurete, compteresse de la .VIIe. nouvelle, seoit pres du jouvencel Philostrate.

$$
\text { (Ibid., p. 91) }
$$

Si l'on inclut (2 b), où la relative qui seoit remplace le gérondif sedendo du textesource, la première version française propose une traduction littérale pour les quatre passages, puisque sedere y est systématiquement traduit par seoir.

Antoine Sabatier de Castres s'autorise à supprimer la cornice (ces passages n’ont donc pas été traduits), mais les traducteurs des $\mathrm{XX}^{\mathrm{e}}$ et $\mathrm{XXI}^{\mathrm{e}}$ siècles nous proposent les versions suivantes :

(2 c) [...] C'est à Néifile, assise à côté du narrateur, que la reine demande de prendre la parole, et de poursuivre la série des plaisantes histoires.

$$
\text { (Jean Bourciez, op. cit., p. 39) }
$$

(3 c) Filomène, qui avait fourni sa tâche, faisait silence. Assis à côté, Dionée n’attendit pas une autre invitation de la reine.

$$
\text { (Ibid., p. 4) }
$$

(4 c) Emilie était assise à côté de Fiammette. Sur un signe de la reine, elle entra franchement dans le jeu.

$$
\text { (Ibid., p. 52) }
$$

(5 c) Assise à côté de Filostrate, Laurette entendit les éloges qu'on faisait de l'astucieux Bergamin.

$$
\text { (Ibid., p. 59) }
$$

(2 d) Le récit de Panfilo suscita par endroits les rires des dames qui le louèrent dans son ensemble; mais la nouvelle attentivement écoutée étant parvenue à sa fin, la reine demanda à Neifile, assise à côté du premier narrateur, de prendre à son tour la parole, afin de poursuivre les réjouissances dans l'ordre jusqu'ici respecté.

(Christian Bec, op. cit., p. 71)

(3 d) Filomena se taisait, s'étant acquittée de sa nouvelle, quand Dioneo qui était assis à côté d'elle et savait, compte tenu des dispositions retenues au départ, que son tour était venu de prendre la parole, sans attendre que la reine le lui ordonne, commença son récit en ces termes: [...]. 
(4 d) Toute l'assemblée étant tombée d'accord pour louer la noble vertu et l'aimable châtiment que la marquise avait infligé au roi de France, comme il plut à la reine, Emilia qui était assise à côté de Fiammetta commença hardiment son récit en ces termes : [...].

(Ibid., p. 86)

(5 d) Filostrato avait, assise à ses côtés, Lauretta qui ayant entendu louer le savoir faire de Bergamino et comprenant que son tour était venu, prit la parole sans attendre qu'on l'en priât et commença plaisamment en ces termes : [...].

$$
\text { (Ibid., p. 94) }
$$

(2 e) La nouvelle de Pamphile, qui parfois fit sourire, fut louée en entier par les dames; lorsqu'on l'eut attentivement écoutée, que son récit fut achevé, la reine commanda à Néiphile, assise près de Pamphile, d'en dire une à son tour, et de suivre ainsi l'ordre du passetemps qui avait débuté.

(Giovanni Clerico, op. cit., p. 75)

(3 e) Déjà se taisait Philomène, qui s'était acquittée de sa nouvelle, lorsque Dionée, assis à côté d'elle, sans attendre d'autre commandement de la reine, puisqu'il savait déjà du fait de l'ordre adopté que c'était son tour de conter, commença de parler de la sorte $[\ldots]$.

(Ibid., p. 83)

(4 e) Émilie, qui était assise à côté de Flammette, après que toutes les dames eurent célébré la valeur de la marquise et l'élégance du châtiment qu'elle avait infligé au roi de France, Émilie, donc, lorsque tel fut le plaisir de sa reine, s'engagea hardimment dans son récit $[\ldots]$.

(Ibid., p. 91)

(5 e) Assise auprès de Philostrate était Laurette, et, quand elle eut entendu célébrer l'adresse de Bergamin, voyant qu'il lui appartenait de dire à présent quelque chose, elle n'attendit aucun commandement et se mit tout aimable à parler de la sorte [...].

$$
\text { (Ibid., p. 99) }
$$

En apposition, en proposition participiale absolue ou introduite par une relative et accompagnée du verbe être, la forme verbale participiale assis(e) se retrouve dans tous ces exemples. Il n'y est nulle part question de seoir, mais de l'adjectif participial d'asseoir : assis.

Là encore, il y a donc incontestablement vieillissement de la version de Premierfait par rapport à toutes les autres, qui présentent une certaine 
homogénéité, probablement par leur volonté de «modernisation ». En effet, seoir, certes peu usité et vieilli, mais tout de même partie intégrante de la langue française, aurait pu y avoir droit de cité.

\section{III.2 Giacere}

\section{III.2.1 'Etre en position horizontale'}

Dans la deuxième nouvelle de la troisième journée, un palefrenier entretient une relation intime avec la femme d'un roi. Ce dernier s'en aperçoit puis se rend dans le lieu où dorment tous les palefreniers pour raser la tête de celui qui lui semble ne pas dormir. Le palefrenier coupable décide alors de raser la tête de tous ses collègues, afin de ne pas être reconnu le lendemain :

(6 a) Costui, che tutto ciò sentito avea, sí come colui che malizioso era, chiaramente savvisò per che cosi segnato era stato; là onde egli, senza alcuno aspettar, si levò, e, trovato un paio di forbicette, delle quali per avventura v'erano alcun paio per la stalla per lo servigio de' cavalli, pianamente andando, a quanti in quella casa ne giacevano, a tutti in simil maniera sopra l'orecchie tagliò i capelli $[. .$.$] .$

(Giovanni Boccaccio, op. cit., p. 190)

(6 b) Pour tant il, sanz plus attendre, se leva de son lit et trouva unes forceps, qui d'aventure estoient en l'estable au service des chevaulx. Si ala secretemeant aux litz de tous le varletz qui en la maison dessudicte estoient endormis et en tel lieu du chief, scestassavoir au dessus les oreilles, il tondit les cheveulx d'un chascun varlet.

(Laurent de Premierfait, op. cit., pp. 316-317)

(6 c) [...] puis, parcourant à son tour les lits de tous ses camarades, il leur coupe tout doucement le même côté de cheveux que le roi lui avait coupé, et s'en retourne dans son lit sans avoir éveillé personne.

(Antoine Sabatier de Castres, op. cit., p. 213)

(6 d) [...] puis il s’approche à pas feutrés de tous les dormeurs de la salle.

(Jean Bourciez, op. cit., p. 190)

(6 e) [...] il se rendit tout doucement auprès de tous ceux qui couchaient dans ce bâtiment $[\ldots]$.

(Christian Bec, op. cit., p. 244)

(6 f) [...] il alla tout doucement auprès de tous ceux qui étaient couchés dans ce bâtiment, et coupa les cheveux à chacun d'eux de la même manière au-dessus de l'oreille [...]. 
L'on n'a ici à aucun moment de traduction littérale. De même, aucun des cinq traducteurs n'a employé le même terme. Deux (le plus ancien et le plus récent) recourent à une forme composée (estre endormi et être couché), deux autres procèdent à une transposition par nominalisation (dormeurllit). Deux seulement choisissent un équivalent orthonymique de giacere, à savoir coucher. Mais si Bourciez choisit simplement coucher, Clerico trouve plus spontané de traduire giacere par être couché. Peut-être peut-on y voir le reflet d'un locuteur $\mathrm{du} \mathrm{XXI}^{\mathrm{e}}$ siècle, qui dirait plus spontanément être couché que coucher pour se référer à la situation d'expérience d'un dormeur. Cela nous renvoie aux découpages chronologiques qui s'instituent différemment entre deux systèmes linguistiques, et au fait que les orthonymes de sedere et giacere sont en français contemporain des lexies composées de deux unités : être assis et être couché, là où un état plus ancien de langue pouvait leur opposer seoir et gésir.

Toutefois, malgré la diversité des solutions adoptées, et ce dès Laurent de Premierfait, on observe dans ces traductions une tendance à l'amplification, que Marie-France Delport définit ainsi : « [Le traducteur] ne se contraint pas à puiser dans les éléments potentiellement inclus dans la situation qu'évoque le textesource. Il ajoute, développe, au gré de ce que son imagination lui présente ; il n'est soumis [...] qu'à une seule restriction : la précision, l'explication apportées doivent être compatibles avec la situation évoquée par la phrase à traduire. $»^{10}$

Cette remarque vaut particulièrement pour les cas que nous appelons de " nominalisation »: les vocables dormeur et lit renvoient nécessairement à la situation de somnolence des êtres instanciant le sujet grammatical. L'adjectif participial endormi de Premierfait nous renseigne également sur cette volonté du traducteur de charger sémantiquement un énoncé, afin de bien "se faire comprendre ". Clerico, plus pragmatique, se contente de l'adjectif participial couché en fonction d'attribut de être : c'est là peut-être l'équivalent référentiel de giacere ici. Dans cette nouvelle, le contexte nous informe que les autres palefreniers sont en train de dormir, et giacere sert juste à déclarer leur existence à cet instant : ce sont des êtres positionnés horizontalement, ils sont donc couchés. Le dernier traducteur en date du Décaméron n'en écrit ni plus, ni moins.

Pour un malade, les choses ne trouvent pas consensus non plus :

(7 a) E un giorno, assai vicini della camera nella quale ser Ciappelletto giaceva infermo, seco medesmi cominciarono a ragionare [...]. Ser Ciappelletto, il quale, come dicemmo, presso giacea là, dove costoro cosí ragionavano, avendo l'udire sottile, sí come le piú volte veggiamo aver gl'infermi, udí ciò che costoro di lui dicevano [...].

(Giovanni Boccaccio, op. cit., p. 49)

(7 b) [...] les deux freres usuriers [...] estans pres du lieu ou Capellet gisoit malade en une chambre, commencerent entre eulx mesmes parler [...]. Capellet donques qui,

10. Marie-France Delport, "Le traducteur omniscient. (Deux figures de traduction : l'explicitation et l'amplification) ", dans Jean-Claude Chevalier, Marie-France Delport, L'horlogerie de Saint Jérôme. Problèmes linguistiques de la traduction, Paris, L'Harmattan, 1995, p. 48. 
comme dict est, gisoit malade pres du lieu ou ces deux freres ainsi parloient ensamble et il ayant le oyemant plus agu, comme l'on <voit> souvant avoir aux malades, il ouy tout ce que ces deux freres usuriers disoient de lui.

(Laurent de Premierfait, op. cit., pp. 39-40)

(7 c) «Que ferons-nous de cet homme? » se disaient-ils l'un à l'autre dans une chambre assez voisine de celle de Chappellet [...]. Maître Chappellet, qui, comme on le voit dans la plupart des malades, avait l'ouïe fine et subtile, ne perdit pas un mot de cette conversation.

(Antoine Sabatier de Castres, op. cit., pp. 31-32)

(7 d) Un jour, tout près de la chambre où Ciappelletto, malade, était couché, ils se mirent à parler entre eux [...]. Ciappelletto, nous l'avons dit[,] était couché près du lieu où discouraient nos gens. Il avait l'ouïe très fine, comme c'est en général le cas des malades.

(Jean Bourciez, op. cit., pp. 30- 31)

(7 e) Un beau jour ils se mirent à converser entre eux dans une pièce toute proche de la chambre où maître Ciappelletto gisait malade [...]. Maître Ciappelletto qui, comme nous le disions, gisait non loin de l'endroit où les frères discutaient, ayant l'ouïe fine, ce qui est souvent le cas des malades, entendit leurs propos sur son compte [...].

(Christian Bec, op. cit., pp. 60- 61)

(7 f) Un jour qu'ils étaient tout près de la chambre où, malade, sire Chapelet reposait, ils se mirent à parler de lui entre eux [...]. Sire Chapelet, qui reposait, comme nous l'avons dit, non loin de l'endroit où ces deux-là devisaient de la sorte, et qui avait l'ouie fine, ainsi qu'il est fréquent de le voir chez les malades, entendit ce que ses hôtes disaient de lui.

(Giovanni Clerico, op. cit., pp. 64- 65)

Gésir deux fois à cinq siècles d'intervalle, être couché une fois, reposer une fois. Sauf chez Sabatier de Castres, qui procède à une nominalisation - celle pour la chambre - et à une suppression, partout, l'accent est mis sur la position horizontale, sur le résultat de l'événement, qui affecte donc l'être pris pour support du verbe, et sur l'inactivité de ce même être. Mais les verbes ne sont pas les mêmes. Sans doute pour sa couleur quelque peu désuète, gésir a été rejeté dans trois traductions, bien que la première acception de gésir dans le TLFI soit : "Être étendu, couché, sans pouvoir se mouvoir (par suite d'un malaise, d'une blessure, d'une maladie, ou de la mort) $"^{11}$. Il semble alors compréhensible que

11. Cf. Jacques Dendien, Le Trésor de la Langue Française Informatisé (TLFI) [En ligne]. 
gésir ait resurgi dans la traduction dirigée par Christian Bec. Il s'agit là d'un choix éditorial, qui consiste à garder le style littéraire de Boccace, avec peut-être une tonalité subrepticement archaïsante, que Bourciez et Clerico ont récusée au profit de termes plus neutres et, partant, plus " modernes $»^{12}$. Ce choix de Bec parait tout à fait légitime, à la lumière de ce qu'affirme Valéry Larbaud : "En réalité, le seul archaïsme qui soit en toute circonstance supportable est celui qui ne se fait pas remarquer, celui que le lecteur, non ou mal instruit du passé de la langue, ou bien n'aperçoit pas, ou bien prend pour une invention de l'auteur, une trouvaille dont il pensera même que c'est un mot ou un tour "bien moderne". "13

\section{III.2.2 'Avoir des relations sexuelles'}

Giacere sert également à exprimer le fait d'avoir un rapport sexuel. Les nombreux exemples du recueil sont, à notre connaissance, systématiquement traduits par coucher, mais l'un d'eux attire particulièrement notre attention. Il s'agit d'un dialogue entre une femme qui affirme tromper son mari avec un prêtre et ce même mari, déguisé en prêtre, qui la confesse :

(8 a) [...] stando adunque fermo domandò la donna: -E come? Non giace vostro marito con voi?

La donna rispose: - Messer si.

- Adunque-disse I geloso- come vi puote anche il prete giacere?

(Giovanni Boccacio, op. cit., p. 437)

(8 b) Le jaloux donques, comme dit est, desguisez en habit de prestre, se maintint ferme et interrogea sa femme :-Commant? Dist il, vostre mari ne gist il pas avec vous? Et elle lui respondit: Ouyl.

Adonc dist le jaloux : - Et commant peuest le prestre chaque nuict coucher avec vous?

(Laurent de Premierfait, op. cit., p. 795)

(8 c) "Mais quoi ! lui dit-il, votre mari ne couche-t-il pas avec vous ? [...] "-Il y couche, mon père. - Comment donc le prêtre peut-il y coucher ? [...]"

(Antoine Sabatier de Castres, op. cit., p. 510)

Jean-Marie Pierrel, CNRS, ATILF, date d'édition ou de mise à jour inconnue [Page consultée le 15/09/2011]. Disponible sur : http://atilf.atilf.fr/tlf.htm

12. Il nous semble qu'à la différence de Jean Bourciez, avec reposer, Giovanni Clerico, quoique par amplification (ajout du sème /maladie/), parvient d'ailleurs à transposer intactes la forme (synthétique) et la valence (monovalence) tout en «modernisant ».

13. Valéry Larbaud, Sous l'invocation de saint Jérôme, Paris, Gallimard [1946], 1997, pp. 123-133. 
(8 d) Mais il [le jaloux] tint bon, pour demander à la pénitente :

-Comment ça, votre mari ne couche pas avec vous?

- Si, messire.

-Alors, comment le prêtre peut-il coucher aussi dans votre lit?

(Jean Bourciez, op. cit., p. 461)

(8 e) [...] il persista donc et demanda sans perdre son sang froid : "Mais comment?

Votre mari ne couche pas avec vous?"

La femme lui répondit : "Mais si, mon frère."

-Alors, rétorqua le jaloux, comment pouvez-vous coucher avec les deux à la fois?

(Christian Bec, opt. cit., p. 562)

(8 f) Donc, tenant ferme, il interrogea la femme :

"Mais comment? Votre mari ne couche-t-il pas avec vous?"

"Si, messire", répondit la dame.

"Alors? dit le jaloux, comment le prêtre peut-il y coucher aussi? "

(Giovanni Clerico, op. cit., p. 589)

Hormis pour Premierfait, coucher l'emporte à l'unanimité. Ce qui frappe, c'est que là où le texte-source présente la même forme (giacere), on trouve sous la plume de Premierfait gésir en alternance avec coucher. S'agissant de la même référence expérientielle, la variation, dans la bouche du même personnage à deux répliques d'intervalle, a de quoi surprendre. Mais elle indique bien que le choix, là aussi, a disparu en français au cours du temps, ou du moins que gésir, ayant perdu en extension, est passé à un registre bien plus recherché (et donc bien moins orthonymique).

\section{III.3 Stare}

\section{III.3.1 'être en position verticale'}

(9 a) [...] e rimase tutto ignudo, e cosi ancora fece la fanciulla; e posesi ginocchione a guisa che adorar volesse e dirimpetto a sé fece star lei.

(Giovanni Boccacio, op. cit., p. 248)

(9 b) [...] et [Rustique] demoura tout nu ; et la pucelle memesmant se despouilla, ainsi comme l'ermite avoit fait. Il adonc se agenouilla devant elle ainsi comme se il voulust icelle adourer; puis fist ester devant soy la pucelle.

(Laurent de Premierfait, op. cit., p. 427)

(9 c) Quand ils sont tout nus l'un et l'autre, Rustique se met à genoux, et fait placer la pauvre innocente vis-à-vis de lui, dans la même situation. 
(9 d) Il reste nu, la néophyte également. Puis il se mit à genou, comme pour prier, et commanda à la jeune fille de prendre, en face de lui, la même posture.

(Jean Bourciez, op. cit., pp. 253-254)

(9 e) [...] il commença à se dépouiller des quelques vêtements qu'il portait et resta tout nu, et la jeunne enfant fit de même ; puis il se mit à genoux en position d'adoration pour ainsi dire et lui dit de se tenir face à lui.

(Christian Bec, op. cit., p. 318)

(9 f) Et il commença à se dépouiller du peu de vêtements qu'il avait, et demeura tout nu, et l'enfant fit de même ; puis il se mit à genoux, comme pour adorer, et la fit se tenir vis-à-vis de lui.

(Giovanni Clerico, op. cit., p. 336)

Ici, il nous semble que l'on voit clairement l'évolution du français à travers les âges. En (9 b), ester est le verbe en vigueur pour déclarer l'existence d'un être en position debout ou verticale. Les deux dernières versions nous proposent, comme pour de très nombreux autres exemples du même type, le verbe pronominal se tenir, indiquant lui aussi une posture physique. Ester apparaîtrait dans cette version résolument archä̈que, voire incompréhensible à un lecteur peu connaisseur de l'histoire de la langue française. On remarquera que Sabatier de Castres traduit stare par placer, et que Bourciez opte pour prendre. L'on passe alors de l'intransitivité à la transitivité. En outre, si pour Sabatier de Castre, l'on est face à un cas d'amplification (placer et situation nous parlent tous deux d'une posture physique), pour Bourciez, il semble se produire un phénomène de compensation. Posture contient en effet le sème / position corporelle dans l'espacel qui manque à prendre. En revanche, se tenir, que l'on voit apparaître dans les deux traductions " nouvelles ", relève plus de ce qu'Umberto Eco nomme négociation, c'est-à-dire " un processo in base al quale, per ottenere qualcosa, si rinuncia a qualcosa d'altro - e alla fine le parti in gioco dovrebbero uscirne con un senso di ragionevole e reciproca soddisfazione alla luce dell'aureo principio per cui non si può avere tutto $"^{14}$.

Négociation, parce que le système linguistique contemporain ne possède plus de forme synthétique qui, comme ester, dit de quelqu'un qu'il est dans une certaine position, et que cette position est verticale.

Toutefois, le point commun de toutes ces traductions est bel et bien la notion de posture physique du sujet ou de l'objet.

14. Umberto Eco, Dire quasi la stessa cosa, Milan, Bompiani, 2003, p. 18. Traduction de l'italien : " un processus selon lequel, pour obtenir quelque chose, on renonce à autre chose d'autre et d'où, au final, les parties en jeu sortent avec un sentiment de satisfaction raisonnable et réciproque, à la lumière du principe d'or selon lequel on ne pas tout avoir »; cf. Myriem Bouzaher (trad.), Dire presque la même chose, Paris, Librairie Générale Française, coll. « Le Livre de Poche ", 2010, p. 19. 


\section{III.3.2 'être en position stationnaire'}

(10 a) E avendo seco stesso diliberato di dover la notte vegnente star presso all'uscio della via ad aspettare se il preste venisse, [i]l geloso, dolente e senza cena, morendo di freddo, quasi tutta la notte stette con le sue armi allato all'uscio ad aspettare se il prete venisse [...].

(Boccaccio, op. cit., pp. 437-438)

(10 b) Et [...] le jaloux ot avec soy deliberé de soy mettre et ester emprés l'uiz de sa chambre en la nuict ensuivant pour attendre se le prestre venroit [...]. Mais le jaloux, courroucié et qui pas soupé n'avoit, et qui estoit comme mort de faim et de froit, et qui par toute nuict a tout ses armes avoit esté pres de l'uiz des degrez en attendanent se le prestre venroit, (...)

(Laurent de Premierfait, op. cit., p. 779-798)

(10 c) Quoiqu'il fit tout son possible pour n'en rien donner à connaitre, il résolut de faire sentinelle, la nuit suivante, dans un réduit voisin de la porte de la rue, pour voir si le prêtre entrerait [...]. Le jaloux, armé de pied en cap, mourant de dépit, de froid et de faim, car il n'avait point soupé, fit le guet jusqu'à ce que le jour parût, et n'ayant pas vu venir le prêtre, il se coucha sur un pliant qu'il y avait dans cette espèce de loge.

(Antoine Sabatier de Castres, op. cit., pp. 511-512)

(10 d) Il résolut de passer la nuit suivante près de la porte de la rue, et d'y attendre la venue du prêtre [...]. Pendant ce temps, contrit, le ventre creux, claquant des dents, le cornard, sous les armes, passa presque toute la nuit près de la porte, dans l'attente du prêtre.

(Jean Bourciez, op. cit., pp. 462-463)

(10 e) Ayant décidé en lui-même de passer la nuit suivante près de la porte donnant sur la rue et d'attendre pour voir si le prêtre viendrait, il dit à sa femme : "Ce soir je dîne et je couche dehors [...].” [...] Le jaloux, affligé, affamé et glacé, passa presque toute la nuit à côté de la porte, les armes à la main, attendant de voir si le prêtre viendrait $[\ldots]$.

(Christian Bec, op. cit., p. 564)

(10 f) S'étant déterminé à se tenir posté, la nuit suivante, près de la porte qui donnait sur la rue, afin de voir si le prêtre viendrait, il dit à sa dame :

"Il faut que j'aille ce soir souper et coucher ailleurs [...]."

Penaud et privé de souper, le jaloux, qui mourait de froid demeura presque toute la nuit avec ses armes à côté de la porte, dans l'attente de la venue du prêtre [...].

(Giovanni Clerico, op. cit., pp. 590-591)

De monoactanciels chez Boccace et Premierfait, on passe aux verbes biactanciels faire, puis passer, avant de revenir, avec Clerico, à se tenir et demeurer. Clerico, par sa traduction " nouvelle ", se rapproche donc le plus du sens originel du texte-source. À partir du moment où ester n'a plus fait partie de la Langue, les traducteurs ont recouru à ces verbes biactanciels qui, par définition, renferment 
en eux deux instants théoriques. Cette répartition de la conceptualisation temporelle se retrouve dans les verbes stare et ester - qui vit toujours de manière cachée dans certaines formes du paradigme de être - : contrairement aux dérivés romans de esse, ils offrent de l'existence une représentation statique, ainsi que l'a démontré Marie-France Delport au sujet du doublet espagnol serl estar : " de cette existence ser propose une représentation thétique, logée [...] dans un seul instant théorique, sans considération d'aucun avant ni d'aucun après, une unité semio-temporelle ; estar en propose, lui, une représentation statique, développée sur deux unités sémio-temporelles de même contenu. ${ }^{15}$

Cette représentation statique se laisse aisément percevoir dans d'autres choix encore, qu'il s'agisse des verbes demourer, continuer, suivre, passer, ou même dans le mot passe-temps :

(11 a) E in questa maniera stettero tanto che tempo parve alla reina d'andare a dormire $[\ldots]$.

(Giovanni Boccaccio, op. cit., p. 44)

(11 b) Et en tel esbannoy demourerent jusques a ce qu'il sambla a la royne que il fesust temps d'aller dormir aprés disner.

(Laurent de Premierfait, op. cit., p. 33)

(11 c) La Reine et toute la compagnie dansèrent au son de ces instruments. Le chant suivit la danse, jusqu’à ce que Pampinée jugea à propos de se reposer.

(Antoine Sabatier de Castres, op. cit., p. 27)

(11 d) Ce passe-temps dura jusqu'à l'heure qui parut à la reine celle du repos.

(Jean Bourciez, op. cit., p. 24)

(11 e) Et l'on continua de cette façon jusqu'à ce qu'il parût temps à la reine d'aller dormir.

(Christian Bec, op. cit., p. 55)

(11 f) Et ils passèrent ainsi le temps jusqu'au moment où la reine jugea qu'il convenait d'aller dormir $[\ldots]$.

(Giovanni Clerico, op. cit., p. 58)

15. Marie-France Delport, "Voix et aspect. La contribution de ser et du "participe" en espagnol médiéval et moderne ", dans Antoine Resano (dir.), Linguistique hispanique. Nantes, 1998. Actes du VII colloque de linguistique hispanique, Nantes, 3, 4 et 5 mars 1998, Université de Nantes, CRINI, 2000, p. 373. 


\section{RÉFÉRENCE ET CONTRAINTES DIACHRONIQUES}

Après examen des rendus français à diverses époques, nous pouvons esquisser un inventaire des capacités référentielles qu'autorisent les trois verbes de position italiens, et qui concernent chacune l'être affecté par l'opération verbale.

Quelle que soit la synchronie, l'on peut déduire que le verbe sedere ne peut contribuer qu'à l'expression d'une seule grande capacité référentielle :

1) se trouver en position assise [seoir, être assis], tandis que celles de stare sont au nombre de trois :

1) posture physique déterminée [ester, se tenir, être debout, etc.] ;

2) position fixe dans l'espace phénoménal ou notionnel [là, placer, se trouver, séjourner, etc.] ;

3) existence (active ou inactive) réitérée par le locuteur [rester, demeurer, prendre, laisser, garder, paix, adj. participial été, état, flotter, se sentir, mener], situation pouvant se manifester syntaxiquement par une tournure intransitive ou transitive.

Certes, il s'agit de trois situations référentielles distinctes mais elles ont toutes en commun le fait d'englober deux instants théoriques, comme le dit probablement le signifié de stare, d'ester et de certaines flexions de être, mais aussi des verbes élus par les traducteurs en remplacement de stare. La possibilité de rendre un verbe intransitif par une tournure transitive n'est certainement pas sans rapport avec cette réitération d'existence citée en situation 3).

Il nous a également semblé pouvoir relever trois grandes capacités référentielles pour giacere:

1) déclaration d'existence d'un être $E$ entretenant des relations sexuelles avec un être E' [gésir, coucher] ;

2) existence en état de somnolence [dormeur, lit, etc.] ;

3) posture physique horizontale et immobile du sujet dans l'espace phénoménal [coucher, être allongé, dormeur, gésir malade].

La compréhension de gésir ne semble pas inclure la première capacité référentielle, ni à date ancienne ni à notre époque, comme le prouve ce passage, que Laurent de Premierfait traduit par deux verbes différents (dormir/gésir) :

(12 a) E avendo sentito che il marito di lei, quantunque di buona famiglia fosse, era avarissimo e cattivo, con lui compose di dovergli dare cinquecento fiorin d'oro, ed egli una notte con la moglie il lasciasse giacere; per che, fatti dorare popolini d'ariento, che allora si spendevano, giaciuto con la moglie, come che contro al piacer di lei fosse, gliele diede.

(Giovanni Boccaccio, op. cit., p. 390)

(12 b) [...] Cestui Degon composa e convint avec le mari que il auroit cinquante florins d'or et que il souffrist dormir le chevalier par toute une nuict avec sa femme. 
Si fist dorer cinquante gros d'argent, qui lors couroient a Florence. Et aprés ce qu'il ot geu avec la femme, toutevoies malgré elle, le chevalier lui donna les cinquante gros dorez $[\ldots]$.

(Laurent de Premierfait, op. cit., p. 712)

\section{Conclusion}

L'examen rapide de ces divers procédés de traduction nous renseigne peutêtre sur les opérations sous-jacentes que tout traducteur de l'italien maitrise de façon plus ou moins exhaustive et inconsciente. Pour un même verbe, les diverses solutions sont tantôt libres, tantôt contraintes.

Au niveau des choix rédactionnels et éditoriaux, nous pouvons affirmer que la traduction de Premierfait, de par le système même du moyen-français, est résolument archaïque et dépassée. Si la belle infidèle de Sabatier de Castres pouvait apparaitre moderne en son temps, elle peut être vue comme vieillie aujourd'hui : la mode n'est en effet plus à l'infidélité. Clerico semble, par des solutions qui ont pris moins de détours, se rapprocher plus du texte-source tout en employant des verbes "modernes ", et conserver la part d'implicite inhérente aux signes choisis par Boccace. Il s'en dégage, à nos yeux, un certain équilibre entre respect de l'époque passée et destinataire contemporain, comme le traducteur en a lui même exprimé le vœu : " Je me suis efforcé de réduire le grand écart entre le point de vue de Boccace sur les choses de ce monde et le nôtre, quand il n'y a pas lieu d'actualiser la perception qu'il a de son environnement. ${ }^{16} \mathrm{Il}$ n'en reste pas moins qu'entre les trois traductions les plus récentes, pour des choix que l'on pourrait parfois juger contestables, ceux-ci ont pris appui sur leurs prédécesseurs, malgré l'épithète "nouvelle » accolée à leur traduction.

Cependant, du fait même de l'obsolescence d'un système linguistique par rapport à un autre, les traductions dites "nouvelles " des œuvres anciennes semblent nécessaires pour laisser intacte, tant que faire se peut, l'absence de distance temporelle qui caractérisait l'écrivain et les lecteurs de l'époque. Les archaïsmes voulus apparaissent alors peu justifiables, comme l'affirme JeanClaude Chevalier :

[...] dans l'original, ce ne sont pas les héros et leurs aventures seuls qui sont du passé, mais aussi le romancier et sa langue. Et [...] ce partage [...] également est à traduire, c'està-dire à conserver. En ne laissant aux siècles révolus que les personnages, les événements où ils trempent et les objets dont ils sont entourés, en transportant en sa personne le romancier dans le temps de nouveaux lecteurs, [le traducteur] se condamne en somme à ne faire qu'un "roman historique". ${ }^{17}$

16. Giovanni Clerico, "Du Décaméron de Giovanni Boccaccio au(x) Décaméron(s) de Jean Boccace ", dans Giovanni Clerico (trad.), Le Décaméron, Paris, Gallimard, 2006, pp. 919.

17. Jean-Claude Chevalier, "La traduction des œuvres anciennes », dans Rosanna Brusegan, Michele A. Cortelazzo (dir.), Il tempo, i tempi-Omaggio a Lorenzo Renzi, Esedra Editrice, 1999, 
Ce qui, à nos yeux, vieillit, c'est le regard que les usagers ou professionnels de la Langue portent sur le signe, y apportant des modifications ci et là dans un souci de plus grande congruence entre ce qu'ils souhaitent exprimer du monde et ce même monde. Ce qui ne vieillit pas, donc, c'est le signifié attaché à chaque signifiant. Apte à laisser s'exprimer de nombreuses situations référentielles en Discours, ce signifié renferme la carte d'identité immuable du verbe. Comme on le voit, pour une même situation référentielle, divers choix de traduction sont adoptés. Mais ces choix, bien que constitués d'une sémiologie différente, présentent tous des similitudes dans leur signifié. Signifié qu'il s'agirait de cerner pour une meilleure compréhension de l'exercice de la traduction et pour faire le départ entre ce qui revient au vieillissement de la référence et ce qui est inhérent à la panchronie d'une langue donnée.

\section{Corpus}

\section{VI.1 Version originale}

Boccaccio Giovanni (c. 1349-1351), Decameron (dir. Cesare Segre), Milan, Mursia, $1966,702 \mathrm{p}$.

\section{VI.2 Traductions françaises du Décaméron [par ordre chronologique]}

Di Stefano Giuseppe (dir.), Premierfait (de) Laurent (trad.), Boccace, Décaméron, traduction de Laurent de Premierfait (1411-1414), Montréal, CERES, 1998, 1240 p.

Bourciez Jean (trad.), Le Décaméron. Traduction nouvelle de Jean Bourciez, Paris, Garnier Frères, 1952, $730 \mathrm{p}$.

Sabatier de Castres Antoine (trad.), Le Décaméron (1779), groupe Ebooks libres et gratuits, $2007,780 \mathrm{p}$.

Bec Christian (dir.), Dozon Marthe (trad.), Guimbard Catherine (trad.), Scialom Marc (trad.), Décaméron, Paris, Librairie Générale Française, coll. « Le Livre de Poche », $1994,896 \mathrm{p}$.

Clerico Giovanni (trad.), Le Décaméron, Paris, Gallimard, 2006, 1058 p.

pp. 51-61 ; maintenant dans Jean-Claude Chevalier, Marie-France Delport, Les Jérômiades. Problèmes linguistiques de la traduction, II, Paris, L'Harmattan, 2010, p. 124. C'est nous qui soulignons. 


\section{Bibliographie}

Bourciez Jean, "Boccace et le Décaméron", in Bourciez Jean (trad.), Le Décaméron. Traduction nouvelle de Jean Bourciez, Paris, Garnier Frères, 1952, pp. I-XII.

Branca Vittore, « Note pour le texte et la traduction », dans Reynard Francisque (trad.), Le Décaméron, Paris, Le club français du livre, pp. XLVII-XLIXI.

Chevalier Jean-Claude, "La traduction des œuvres anciennes ", dans Chevalier JeanClaude, Delport Marie-France (2010), Les Jérômiades. Problèmes linguistiques de la traduction, II, Paris, L'Harmattan, pp. 115-126.

Clerico Giovanni, "Du Décaméron de Giovanni Boccaccio au(x) Décaméron(s) de Jean Boccace ", dans Clerico Giovanni (trad.), Le Décaméron, Paris, Gallimard, 2006, pp. 907-920.

Delport Marie-France, "Les horloges du traducteur ", dans Chevalier Jean-Claude, Delport Marie-France, L’horlogerie de Saint Jérôme. Problèmes linguistiques de la traduction, Paris, L'Harmattan, 1995, pp. 11-26.

- "Le traducteur omniscient. (Deux figures de traduction : l'explicitation et l'amplification) ", dans Chevalier Jean-Claude, Delport Marie-France, L'horlogerie de Saint Jérôme. Problèmes linguistiques de la traduction, Paris, L'Harmattan, 1995, pp. 45-58.

— "Voix et aspect. La contribution de ser et du "participe" en espagnol médiéval et moderne ", dans Resano Antoine (sous la dir. de), Linguistique hispanique. Nantes, 1998. Actes du VIIe colloque de linguistique hispanique, Nantes, 3, 4 et 5 mars 1998, Université de Nantes, CRINI, 2000, pp. 369-380.

Dendien Jacques, le Trésor de la Langue Française informatisé (TLF) [En ligne]. Pierrel Jean-Marie, CNRS, ATILF, date d'édition ou de mise à jour inconnue [Page consultée le 15/09/2011]. Disponible sur : http://atilf.atilf.fr/tlf.htm

Di Stefano Giuseppe, "Introduction ", dans Di Stefano Giuseppe (dir.), Boccace, Décaméron, traduction de Laurent de Premierfait (1411-1414), Montréal, CERES, 1998, pp. IX-XXI.

Eco Umberto, Dire quasi la stessa cosa, Milan, Bompiani, 2003, 398 p.

- Dire presque la même chose, traduit de l'italien par Bouzaher Myriem, Paris, Librairie Générale Française, coll. «Le Livre de Poche », 2010, 503 p.

Larbaud Valéry, Sous l'invocation de saint Jérôme, Paris, Gallimard [1946], 2e éd. 1997, $366 \mathrm{p}$.

Larochette Joe, "Les aspects verbaux en espagnol moderne ", Revue belge de philologie et d'histoire, $\mathrm{n}^{\circ} 23,1944$, pp. 39-72.

Pottier Bernard, Sémantique générale [1992], 2011, Paris, PUF, 242 p. 